REVIEW

\title{
What is quality in surgical pathology?
}

\section{R E Nakhleh}

J Clin Pathol 2006;59:669-672. doi: 10.1136/jcp.2005.031385

Quality in surgical pathology may be defined as accurate, timely, and complete reports. Achieving quality requires substantial investment in the basic structure and in the people who undertake surgical pathology. Quality assurance and improvement works best when it is woven into the systems of surgical pathology with well informed, well trained, and knowledgeable staff.

\section{MEASURING QUALITY IN SURGICAL PATHOLOGY}

Then how do we define quality in surgical pathology? Elements of quality that are important in surgical pathology include report accuracy, timeliness, and report completeness. ${ }^{4}$ In manufacturing, one typically adds price or cost into the expectation of quality. However, in the current system of third party payers in medicine, price has been diminished in consideration of quality.

If it is agreed that accuracy, timeliness, and completeness are desired features in surgical pathology, then designing a quality assurance and improvement (QA\&I) plan should focus on these three elements. Typical quality assurance and improvement plans contain five categories of monitors (table 1)..$^{5-9}$ The first three categories include the entire test cycle (pre-analytic, analytic, and post-analytic phases). A reason for this design is the rationale that defects may occur at any point in the test cycle, resulting in an erroneous diagnosis, so the entire test cycle should be monitored. ${ }^{10}$ Another reason is the desire for subsets of the process to work well together in producing a final product. Therefore, by examining the process in its entirety and in detail one may introduce modifications within the process that ultimately improve the product. The majority of monitors within the three phases of the test cycle are used to assure accuracy, although relatively few are focused on the actual diagnosis. Completeness is usually addressed in post-analytic phase monitors, even though many elements of completeness have their roots in the analytic phase of the test cycle. ${ }^{7}$

The other two categories of monitors are turnaround time (TAT) and customer or clinician satisfaction. Timeliness is entirely addressed by TAT monitors. Customer or clinician satisfaction aims to measure the whole process from the clinician's perspective. The following is a brief discussion of QA\&I plan monitors and how they measure surgical pathology accuracy, timeliness, and completeness.

\section{Pre-analytic phase}

In the pre-analytic phase of the test cycle several elements may be monitored. Patient or specimen identification, however, is the most important of these elements. Mishaps in specimen identification have led to unwarranted procedures and very dramatic headlines in the lay press. ${ }^{11}$ It is critical that a specimen is reported on the correct patient and this begins with specimen labelling

Abbreviations: $\mathrm{CoC}$, Commission on Cancer; FISH fluorescent in situ hybridisation; QA\&l, quality assurance and improvement; TAT, turnaround time
Dr Raouf E Nakhleh, Department of Pathology, 4201 Belfort Rd, St Luke's Hospital/Mayo Clinic Jacksonville, Jacksonville, FL 32216, USA; nakhleh. raouf@mayo.edu is derived from statistical methods at 1000000 product is derived from statistical methods at the six sigma or six standard deviations of liketi in a normal distribution of customers. B comparison, various studies have estimated surgical pathology error rates from as low as $0.25 \%$ (2500 per million) to as high as $40 \%{ }^{4}$ Most investigators, however, agree that a significant error rate in surgical pathology is in the range of $0.5 \%$ to $1 \%$. 


\section{Table 1 Quality assurance and improvement monitors}

\section{Pre-analytic}

Specimen fixation

Specimen delivery

Specimen identification

Adequacy of clinical history

Accessioning errors

\section{Analytic}

Intra-operative

Frozen section - permanent section concordance

Final diagnosis

Peer review error rate

Histology and gross room monitors

Block labelling errors

Slide labelling errors

Slide quality

Immunohistochemistry

Frequency of repeat slides

Annual inventory of antibodies and frequency of use

External validation of selected antibodies

Other ancillary study monitors include monitors for FISH, EM, other

molecular studie

\section{Post-analytic}

Transcription errors

Verification errors

Report delivery errors

Incomplete reports

Diagnostic finding correlation with ancillary studies (IHC, EM, FISH)

Turnaround time (TAT)

Frozen section

Biopsy

Large specimen

Preliminary and final necropsy reports

Clinician satisfaction and/or complaints

Overall satisfaction

Diagnostic accuracy

Frozen section timeliness and accuracy

Report timeliness

Report completeness

Pathologist availability

Recent changes

EM, electron microscopy; FISH, fluorescent in situ hybridisation; IHC, immunohistochemistry.

and accessioning. This process is multifactorial and involves many individuals outside the laboratory. Therefore, significant improvement in specimen identification requires acceptance of this goal across an institution with a substantial awareness campaign and the use of stringent labelling standard..$^{12}$ Fortunately, accrediting bodies such as the Joint Commission for Accreditation of Healthcare Organizations and the College of American Pathologists have made accurate patient identification a cardinal patient safety goal.

Other critical elements in the pre-analytic phase include lost specimens, appropriate fixation, and adequate clinical history $\cdot{ }^{13}$ Most of the elements of the pre-analytic phase aim at improving the accuracy of diagnosis, particularly adequate clinical history. Clinical history has been shown to affect the accuracy and completeness of pathology reports. ${ }^{14}$ Unfortunately, we are unaware of studies that have attempted improvement of adequate clinical history received with specimens.

\section{Analytic phase}

The analytic phase of the test cycle begins with gross examination of the specimen and ends with diagnosis. All manipulations of the specimen subsequent to gross examination, such as histological preparation and immunohistochemistry, that lead to a diagnosis are therefore components of the analytic phase of the test cycle. Most critical in this phase is the act of diagnosis itself. Many elements culminate in a diagnosis, including gross dissection and section, embedding, histological sectioning, staining, special and immunohistochemical staining, possible other ancillary studies, and microscopic interpretation. The accuracy of the final diagnosis is a measure of the effectiveness of all of these sequential steps.

In the absence of a better method, judging the correctness of surgical pathology diagnoses has become an exercise in peer review and may be the most important measure of quality with respect to patient care. ${ }^{15}$ Different methods of peer review have been used (table 2). However, no single method has been shown to be superior in detecting errors. Second review of cases before verification has been shown to reduce the number of amended reports. ${ }^{16}{ }^{17}$ Thus as a preventative measure many institutions have installed second pathologist review before sign-out on selected cases (for example, breast biopsies, pigmented skin lesions).

All measures in the analytic phase aim to improve diagnostic accuracy, including monitors of histology or immunohistochemistry quality as well as other ancillary studies such as in-situ hybridisation. Monitors of immunohistochemistry may become more important in the future, particularly for markers that determine treatment, such as Her $2 /$ neu. $^{18}$

\section{Post-analytic phase}

The post-analytic phase of the test cycle begins with dictation of the gross and microscopic examination and the final diagnosis and includes transcription, report correction, verification, and report delivery. ${ }^{7}$ Keys in the post-analytic phase are accurate transcription, complete reporting, and report delivery. Only recently has the issue of complete reporting in surgical pathology been given sufficient attention. There is a drive toward evidence based medicine. ${ }^{19}$ This is particularly important in oncology, where many protocols are dependent on the pathological staging of tumours. The Cancer Program Standards 2004 publication of the Commission on Cancer ( $\mathrm{CoC}$ ) requires that $90 \%$ of pathology reports that include a cancer diagnosis will contain the scientifically validated data elements outlined on the surgical case summary checklist of the College of American Pathologists (CAP) publication, Reporting on Cancer Specimens..$^{20}{ }^{21}$ The use of summary checklists has been shown to be very effective in providing more complete reports. ${ }^{22}$ In view of the $\mathrm{CoC}$ standard, monitoring of report completeness for cancer resection seems necessary and worthwhile. Other situations where report completeness is an issue include CAP standards for the correlation of histopathology with previous cytological or histological material and with various ancillary studies. ${ }^{23}$

As stated above, TAT is a critical element of quality and usually covers all aspects of the laboratory test cycle. While TAT may be fragmented into smaller components, the total TAT is the only measure by which the clinician or customer will judge the laboratory. ${ }^{24}$ Smaller components, however, are important to understand when an intervention is planned to improve the total TAT.

Customer or clinician satisfaction is probably one of the most important measures of quality because it lends insight into the clinician's perception of the laboratory. While there are many elements that when combined add up to a quality laboratory, clinician satisfaction is also based on the additional factor of expectations..$^{25}$ Thus a laboratory may have accurate, timely, and complete reports, yet a clinician may still have the perception of poor quality if they have unrealistic expectation. Therefore, in addition to managing and monitoring all the elements of quality, the pathologist must also manage clinician expectations and make sure that they are realistic. Without some effort to obtain clinician 
Table 2 Different methods of peer review

Review of a randomly selected percentage of cases

Focused internal review of specific organ system or malignancy type (for example, breast cancer)

Interdepartmental conferences (for example, tumour board)

Intradepartmental quality assurance conference

Frozen section/permanent section correlation

Cytology/surgical pathology correlation

Review of previous pathology material

Intradepartmental review of material before release to other institutions.

Review of outside diagnosis of in-house cases

feedback, some problems - at least from the clinicians' perspective-may never be identified.

\section{STRUCTURAL COMPONENT OF A QUALITY SURGICAL PATHOLOGY LABORATORY}

A quality assurance and improvement plan is merely a small component in maintaining a quality laboratory. Quality in a laboratory is dependent on a host of structural and personnel factors that are necessary, regardless of the QA\&I plan. Even better, quality assurance and improvement must be weaved into all the other systems of the laboratory to achieve the absolute best results. The following is a discussion of critical elements needed to maintain a quality laboratory.

\section{Work force}

A flexible, well trained, knowledgeable staff is key to the success of any organisation. This applies to all levels of work within surgical pathology, including pathologists, pathologist's assistants, histology staff, and the secretarial staff. Important aspects in building the staff are qualifications, suitability, sufficient redundancy, and the ability to work with others. ${ }^{27}$ Of course, individuals must have the appropriate qualifications for the jobs they are doing, but more importantly people must be suited to their duties. Individuals with the same qualifications may have vastly different strengths and weaknesses and must be placed in positions to take advantage of their strengths, doing the opposite is a sure recipe for failure. In building a work force, sufficient redundancy in skills is critical to assure continuity or work functions are not affected during an individual's absence. Finally, the staff should work together as a team. The ability to work with others is critical for maintaining a healthy environment and is beneficial to patient safety. ${ }^{28}$

\section{Continuous education and training}

Medical knowledge and treatment is constantly changing. The medical staff must constantly seek out new knowledge and adopt new practices as they become available. These new concepts should be shared and discussed with colleagues, and collectively either adopted or rejected. As individuals are hired they should be trained to the specific peculiarities of their jobs within a particular institution. Individuals should also have regular training in a host of other areas, such as safety and quality improvement, as well as any impending changes in their job duties.

\section{Comprehensive computer system}

A comprehensive computer system can greatly enhance the quality of a surgical pathology laboratory..$^{29}{ }^{30}$ While all the necessary technology is available, comprehensive computer systems are rare. The ideal system has the ability to pull together all the components of surgical pathology with integrated quality assurance and quality control checks. One may envision a system that allowed physician remote order entry so that the clinical history is mandated and specimens are accounted for as they arrive. A comprehensive system would be tied with an institutional database to confirm the patient's identity at accession. Subsequently, blocks and slides would be labelled automatically with the accession number and the patient's name and any other identifying information. The system would provide tracking mechanisms through the use of bar code or similar technology, so that all cases, blocks, slides, and reports are accounted for throughout the process. In addition, bar code technology would be used to input dictation and transcription so that misidentification errors are reduced. A comprehensive computer system could check and alert if reports have incomplete elements or if cases are not completed within a reasonable time. Such a system could then deliver reports electronically to the ordering physician as well as to other venues such as tumour registries. Finally, a comprehensive computer system could generate numerous quality reports in real time and could offer alerts when set parameters are not met.

\section{Standardised tasks and language}

Quality laboratories have set predetermined standardised procedures that are easily accessible and well known by the staff. A key to quality is the elimination of competing procedures. ${ }^{27}$ This is beneficial in reducing confusion over which procedures should be followed, but more importantly it leads to tremendous efficiencies in laboratory operations. Employees must be trained in accepted procedures as they are hired, but also regularly updated as procedures are modified.

Just as important is the standardisation of terms used within the laboratory and in communication with clinicians and physician's offices outside of the laboratory, including diagnostic terminology. Diagnostic terminology is constantly being revised and laboratories must have mechanisms to review and update diagnostic criteria and terminology annually. By the same token, this needs to be communicated to all who are likely to encounter these terms. To reduce confusion and enhance customer satisfaction, clinicians should be included.

\section{The ability to change}

Key to the success of most organisations is their ability to respond to challenges quickly and effectively. Inherent in these organisations is an ability to adapt and change. While the basis of surgical pathology practice has not changed significantly over the past half century, changes in the approach to individual diseases are occurring at a much more rapid pace. Breast cancer is a prime example of this evolution. Thirty years ago a pathology report on a breast cancer included a diagnosis and lymph node status. Today a report should include a diagnosis, tumour grade, tumour size, vascular involvement, lymph node status, margin status, and distance to margin if negative, oestrogen and progesterone receptor status, Her2/neu immunostain, and possibly a FISH result. Along the way several other factors such as flow cytometry and proliferation markers were at one point thought to be important and were included in pathology reports, but have now been shown to be less significant in 
determining outcome or treatment and therefore may not need to be included. Although at variable rates, this type of evolution is occurring in many disease processes. A quality surgical pathology laboratory must remain current with all of these changes and ideally serve as a source of information to clinicians served by that laboratory.

\section{Regulatory compliance}

Finally, it may be superfluous to say that a laboratory must be in regulatory compliance to operate. Of course this is necessary for licensure, but more importantly, regulatory standards are helpful in guiding laboratories to set up policies and procedures. ${ }^{31}$ For the most part regulatory requirements are the minimum standards necessary and serve as a foundation for systems and organisational structures to achieve a higher level of quality.

\section{REFERENCES}

1 The American Heritage ${ }^{\circledR}$ Dictionary of the English Language, 4th edition. Copyright $\odot 2000$, Houghton Mifflin Company.

2 Quality. From Wikipedia, the free encyclopedia, http://en.wikipedia.org/ wiki/Quality.

3 Six Sigma. From Wikipedia, the free encyclopedia, http://en.wikipedia.org/ wiki/Six_Sigma.

4 Nakhleh RE. Introduction. In:Nakhleh RE, Fitzgibbons PL, editors. Quality management in anatomic pathology: promoting patient safety through systems improvement and error reduction. Northfield: The College of American Pathologists, 2005:1-4.

5 Brown RW. Preanalytic variables: specimen submission and handling. In:Nakhleh RE, Fitzgibbons PL, editors. Quality management in anatomic pathology: promoting patient safety through systems improvement and error reduction. Northfield: The College of American Pathologists, 2005:45-9.

6 Weiss MA. Analytic variables: diagnostic accuracy. In:Nakhleh RE, Fitzgibbons $\mathrm{PL}$, editors. Quality management in anatomic pathology: promoting patient safety through systems improvement and error reduction. Northfield: The College of American Pathologists, 2005:50-61.

7 Fitzgibbons PL. Postanalytic variables: report adequacy and integrity. In:Nakhleh RE, Fitzgibbons PL, editors. Quality management in anatomic pathology: promoting patient safety through systems improvement and error reduction. Northfield: The College of American Pathologists, 2005:61-5.

8 Coffin CM, Turn around time. In:, Nakhleh RE, Fitzgibbons PL, editors. Quality management in anatomic pathology: promoting patient safety through systems improvement and error reduction. Northfield: The College of American Pathologists, 2005:65-70.

9 Nakhleh RE, Customer satisfaction. In:, Nakhleh RE, Fitzgibbons PL, editors. Quality management in anatomic pathology: promoting patient safety through systems improvement and error reduction. Northfield: The College of American Pathologists, 2005:70-72.

10 Nakhleh RE. Designing a quality improvement plan, In: Nakhleh RE, Fitzgibbons PL, editors. Quality management in anatomic pathology: promoting patient safety through systems improvement and error reduction. Northfield: The College of American Pathologists, 2005:5-8.

11 Fisher B. U of C Hospital sued for error that resulted in removal of breast. Chicago Sunday Times 2005 May 11.

12 Nakhleh RE. Lost, mislabeled and unsuitable surgical pathology specimens. Pathology Case Reviews 2003;8:98-102.
13 Nakhleh RE, Zarbo RJ. Surgical pathology specimen identification and accessioning: a College of American Pathologists Q-Probes study of $1,004,115$ cases from 417 institutions. Arch Pathol Lab Med $1996 ; 120: 227-33$

14 Nakhleh RE, Gephardt G, Zarbo RJ. Necessity of clinical information in surgical pathology: a College of American Pathologists Q-probes study of 771,475 surgical pathology cases from 341 institutions. Arch Pathol Lab Med 1999; 123:615-19.

15 Zarbo RJ, Meier FA, Raab SS. Error detection in anatomic pathology. Arch Pathol Lab Med 2005;129:1237-45.

16 Nakhleh RE, Zarbo RJ. Amended reports in surgical pathology and implications for diagnostic error detection and avoidance: a College of American Pathologists' Q-probes study of 1,667,547 accessioned cases in 359 laboratories. Arch Pathol Lab Med 1998;22:303-9.

17 Novis DA. Detecting and preventing the occurrence of errors in the practices of laboratory medicine and anatomic pathology: 15 years' experience with the College of American Pathologists' Q-probes and Q-tracks programs. Clin Lab Med 2004;24:965-78.

18 Wick MR, Mills SE. Consensual interpretive guidelines for diagnostic immunohistochemistry. Am J Surg Pathol 2001;25:1208-10.

19 Marchevsky AM, Wick MR. Evidence-based medicine, medical decision analysis, and pathology. Hum Pathol 2004;35:1179-88.

20 Commission on Cancer. Standard 4.6. The guidelines for patient management and treatment currently required by the $\mathrm{CoC}$ are followed. Commission on Cancer of the College of American Surgeons Cancer Program Standards. http://www.facs.org/cancer/coc/standardsclarifications.html.

21 College of American Pathologists. Cancer protocols and checklists. Updated 14 April 2005. http://www.cap.org/apps/docs/cancer_protocols/ protocols_index.html.

22 Branston LK, Greening S, Newcombe RG, et al. The implementation of guidelines and computerized forms improves the completeness of cancer pathology reporting. The CROPS project: a randomized controlled trial in pathology, Eur J Cancer 2002;38:764-72.

23 College of American Pathologists. Laboratory Accreditation Program inspection checklists. Updated 20 October 2005. http://www.cap.org/apps/ docs/laboratory_accreditation/checklists/checklistftp.html.

24 Zarbo RJ, Gephardt GN, Howanitz PJ. Intralaboratory timeliness of surgical pathology reports: results of two College of American Pathologists Q-probes studies of biopsy and complex specimens. Arch Pathol Lab Med 1996; 120:234-44

25 Zarbo RJ, Nakhleh RE, Walsh M. Customer satisfaction in anatomic pathology; a College of American Pathologist Q-probes study of 3065 physician surveys from 94 laboratories. Arch Pathol Lab Med 2003;127:23-9.

26 Nakhleh RE, Jones B, Zarbo RJ. Mammographically directed breast biopsies: a Q-probes study of clinical physician expectations, specimen handling and reporting characteristics in 434 institutions. Arch Pathol Lab Med reporting characteristic

27 Spath PL. Reducing errors through work systems improvement. In:Spath PL, editor. Error reduction in health care. San Francisco: Jossey-Bass, 1999:199-234.

28 Grumbach K, Bodenheimer T. Can healthcare teams improve primary care practice? JAMA 2004;291:1246-51.

29 Bates DW, Cohen M, Leape LL, et al. Reducing the frequency of errors in medicine using information technology. J Am Med Inform Assoc 2001;8:299-308.

30 Bates DW. The quality case for information technology in healthcare. BMC Med Informatics Decision Making 200, 2:7-16.

31 Carter DK, Regulatory compliance. In:, Nakhleh RE, Fitzgibbons PL, editors. Quality management in anatomic pathology: promoting patient safety through systems improvement and error reduction. Northfield: The College of American Pathologists, 2005:9-31. 